\title{
SUPPLEMENT TO: MULTILEVEL MODELS WITH STOCHASTIC VOLATILITY FOR REPEATED CROSS-SECTIONS: AN APPLICATION TO TRIBAL ART PRICES
}

\author{
By Silvia Cagnone ${ }^{\dagger}$, Simone Giannerini ${ }^{\dagger, *}$ and Lucia Modugno ${ }^{\dagger}$ \\ University of Bologna ${ }^{\dagger}$
}

1. Recursive algorithm for computing the likelihood. In order to facilitate the computation of the likelihood approximated with the GaussLegendre quadrature method we use the matrix notation for the recursive algorithm. For simplicity of notation, from now on we omit the conditioning upon the vector of covariates. Define the $\left(n_{u} \times 1\right)$ and $\left(n_{h} \times 1\right)$ vectors of the initial probabilities as

$$
\mathbf{f}_{u_{1}}=\left(f\left(u_{1}^{*}\right), f\left(u_{2}^{*}\right), \ldots, f\left(u_{n_{u}}^{*}\right)\right)^{\prime} \quad \text { and } \quad \mathbf{f}_{h_{1}}=\left(f\left(h_{1}^{*}\right), f\left(h_{2}^{*}\right), \ldots, f\left(h_{n_{h}}^{*}\right)\right)^{\prime}
$$

and the $\left(n_{u} n_{h} \times 1\right)$ vector of the initial conditional probabilities as

$$
\mathbf{f}_{1}=\left(f\left(\mathbf{y}_{1} \mid u_{1}^{*}, h_{1}^{*}\right), \ldots, f\left(\mathbf{y}_{1} \mid u_{n_{u}}^{*}, h_{1}^{*}\right), f\left(\mathbf{y}_{1} \mid u_{1}^{*}, h_{2}^{*}\right), \ldots, f\left(\mathbf{y}_{1} \mid u_{n_{u}}^{*}, h_{n_{h}}^{*}\right)\right)^{\prime} .
$$

In the same way, define the probability transition matrices of the two discretized latent variables as

$\mathbf{F}_{h}=\left[\begin{array}{ccc}f\left(h_{1}^{*} \mid h_{1}^{*}\right) & \ldots & f\left(h_{1}^{*} \mid h_{n_{h}}^{*}\right) \\ \vdots & \vdots & \vdots \\ f\left(h_{n_{h}}^{*} \mid h_{1}^{*}\right) & \ldots & f\left(h_{n_{h}}^{*} \mid h_{n_{h}}^{*}\right)\end{array}\right] ; \mathbf{F}_{u}=\left[\begin{array}{ccc}f\left(u_{1}^{*} \mid u_{1}^{*}\right) & \ldots & f\left(u_{1}^{*} \mid u_{n_{u}}^{*}\right) \\ \vdots & \vdots & \vdots \\ f\left(u_{n_{u}}^{*} \mid u_{1}^{*}\right) & \ldots & f\left(u_{n_{u}}^{*} \mid u_{n_{u}}^{*}\right)\end{array}\right]$

and the $\left(n_{u} n_{h} \times 1\right)$ vector of conditional probabilities for $t=2, \ldots, T$ as

$$
\mathbf{f}_{t}=\left(f\left(\mathbf{y}_{t} \mid u_{1}^{*}, h_{1}^{*}\right), \ldots, f\left(\mathbf{y}_{t} \mid u_{n_{u}}^{*}, h_{1}^{*}\right), f\left(\mathbf{y}_{t} \mid u_{1}^{*}, h_{2}^{*}\right), \ldots, f\left(\mathbf{y}_{t} \mid u_{n_{u}}^{*}, h_{n_{h}}^{*}\right)\right)^{\prime} .
$$

Finally, define the vectors $\mathbf{w}_{u}=\left(w_{u 1}, w_{u 2}, \ldots, w_{u n_{u}}\right)^{\prime}, \mathbf{w}_{h}=\left(w_{h 1}, w_{h 2}, \ldots, w_{h n_{h}}\right)^{\prime}$ and the matrices $\mathbf{W}_{u}=\left(\mathbf{w}_{u}, \mathbf{w}_{u}, \ldots, \mathbf{w}_{u}\right), \mathbf{W}_{h}=\left(\mathbf{w}_{h}, \mathbf{w}_{h}, \ldots, \mathbf{w}_{h}\right)$ of dimensions $\left(n_{u} \times n_{u}\right)$ and $\left(n_{h} \times n_{h}\right)$ respectively. The approximated marginal likelihood can be computed through the following iterative algorithm

${ }^{*}$ Corresponding author.

Keywords and phrases: multilevel model, hedonic regression model, dependent random effects, stochastic volatility, autoregression 
1. compute the joint probability at the first time point $(t=1)$ :

$$
\mathbf{l}_{1}=r_{u} r_{h} \operatorname{diag}\left(\mathbf{f}_{1}\right)\left(\left(\mathbf{f}_{h_{1}} \circ \mathbf{w}_{h}\right) \otimes\left(\mathbf{f}_{u_{1}} \circ \mathbf{w}_{u}\right)\right)
$$

where $\circ$ indicates the Hadamard product between two matrices and $\operatorname{diag}\left(\mathbf{f}_{1}\right)$ is the block-diagonal matrix created from the vector $\mathbf{f}_{1}$. The generic element of $\mathbf{l}_{1}$ is $l_{1 i j}=f\left(\mathbf{y}_{1}, u_{t}=u_{i}^{*}, h_{t}=h_{j}^{*}\right)$.

2. compute the forward intermediate joint probabilities for $t=2, \ldots, T$ :

$$
\mathbf{l}_{t}=r_{u} r_{h} \operatorname{diag}\left(\mathbf{f}_{t}\right)\left(\left(\mathbf{F}_{h} \circ \mathbf{W}_{h}\right) \otimes\left(\mathbf{F}_{u} \circ \mathbf{W}_{u}\right)\right) \mathbf{l}_{t-1},
$$

where $\operatorname{diag}\left(\mathbf{f}_{t}\right)$ is the block-diagonal matrix created from the vector $\mathbf{f}_{t}$. The generic element of $\mathbf{l}_{t}$ is $l_{t i j}=f\left(\mathbf{y}_{1}, \ldots, \mathbf{y}_{t}, u_{t}=u_{i}^{*}, h_{t}=h_{j}^{*}\right)$.

3. compute the approximated marginal likelihood (Eq. 7 of the article) as

$$
\tilde{L}(\boldsymbol{\theta} \mid \mathbf{y}, \mathbf{x})=\mathbf{1}^{\prime} \mathbf{l}_{T}
$$

where $\mathbf{1}$ is a $\left(n_{u} n_{h} \times 1\right)$ vector of ones.

The maximization of expression (3) is carried out by using a quasi-Newton algorithm in which the gradient and the Hessian matrix are obtained through numerical derivatives. The choice of the starting values for $\boldsymbol{\theta}$ requires two separate strategies, one for the parameters of the stochastic volatility component and one for the remaining parameters. As for the former, we adapt to repeated cross-sectional data the method used by Bartolucci and De Luca (2001) in a time series context. First, consider the stochastic volatility model for the error terms of the SVARE model

$$
y_{i t}^{*}=y_{i t}-\beta_{0}-u_{t}-\mathbf{x}_{i t}^{\prime} \boldsymbol{\beta}=\exp \left(h_{t} / 2\right) \epsilon_{i t},
$$

from which, as observed by Harvey et al. (1994), $\log y_{i t}^{* 2}=h_{t}+\log \epsilon_{i t}^{2}$. Since $\mathrm{E}\left(\log \epsilon_{i t}^{2}\right)=-1.27$ we have that

$$
h_{t} \approx h_{t}^{*}=\sum_{i=1}^{n_{t}}\left(\log \hat{y}_{i t}^{* 2}\right) / n_{t}+1.27
$$

where $\hat{y}_{i t}^{*}$ are obtained as the level- 1 residuals of the SVARE model. Second, smooth $h_{t}^{*}$ through a $k$-term moving average, run a regression of $h_{t}^{*}$ on $h_{t-1}^{*}$ and use the resulting estimates as initial values of the stochastic volatility parameters. As concerns the remaining parameters, the estimates of the fitted ARE model are taken as starting values. 
2. Filtering, Smoothing and Prediction. In order to obtain optimal estimators of the unobserved-state vectors $\mathbf{u}$ and $\mathbf{h}$, we perform filtering and smoothing, which differ in the conditioning information set. Moreover, we derive the one-step-ahead predictors.

2.1. Filtering. Denoting with $\mathbf{Y}_{t}=\left(\mathbf{y}_{1}, \ldots, \mathbf{y}_{t}\right)$, the filtered volatility and the filtered random effects at time $t$ are defined as

$$
\begin{aligned}
& \hat{h}_{t}\left(\mathbf{Y}_{t}\right)=E\left(h_{t} \mid \mathbf{Y}_{t}\right)=\int h_{t} f\left(h_{t} \mid \mathbf{Y}_{t}\right) d h_{t} \\
& \hat{u}_{t}\left(\mathbf{Y}_{t}\right)=E\left(u_{t} \mid \mathbf{Y}_{t}\right)=\int u_{t} f\left(u_{t} \mid \mathbf{Y}_{t} d u_{t}\right.
\end{aligned}
$$

The filtered values are computed directly from the quantities obtained in the forward recursive algorithm:

$$
\begin{aligned}
& \hat{h}_{t}\left(\mathbf{Y}_{t}\right)=\int h_{t} \frac{f\left(h_{t}, \mathbf{Y}_{t}\right)}{f\left(\mathbf{Y}_{t}\right)} d h_{t}=\frac{\iint h_{t} f\left(\mathbf{Y}_{t}, u_{t}, h_{t}\right) d u_{t} d h_{t}}{\iint f\left(\mathbf{Y}_{t}, u_{t}, h_{t}\right) d u_{t} d h_{t}} \simeq \frac{\sum_{j}^{n_{h}} h_{j}^{*} \sum_{i}^{n_{u}} l_{t i j}}{\sum_{i}^{n_{u}} \sum_{j}^{n_{h}} l_{t i j}} \\
& \hat{u}_{t}\left(\mathbf{Y}_{t}\right)=\int u_{t} \frac{f\left(u_{t}, \mathbf{Y}_{t}\right)}{f\left(\mathbf{Y}_{t}\right)} d u_{t}=\frac{\iint u_{t} f\left(\mathbf{Y}_{t}, u_{t}, h_{t}\right) d h_{t} d u_{t}}{\iint f\left(\mathbf{Y}_{t}, h_{t}, u_{t}\right) d u_{t} d h_{t}} \simeq \frac{\sum_{i}^{n_{u}} u_{j}^{*} \sum_{j}^{n_{h}} l_{t i j}}{\sum_{i}^{n_{u}} \sum_{j}^{n_{h}} l_{t i j}}
\end{aligned}
$$

2.2. Smoothing. In the multilevel framework, the random effects are predicted by using either the Best Linear Unbiased Predictor or the Empirical Bayes Predictor. In both cases, the best predictor is the expected value conditioned to the whole observed sample or empirical posterior distribution (for an overview see Searle et al. (1992) and Skrondal and Rabe-Hesketh (2004)). In stochastic volatility models the smoothed values are:

$$
\hat{h}_{t}\left(\mathbf{Y}_{T}\right)=E\left(h_{t} \mid \mathbf{Y}_{T}\right)=\iint h_{t} \frac{f\left(\mathbf{Y}_{t}, h_{t}, u_{t}\right) f\left(\mathbf{y}_{t+1}, \ldots, \mathbf{y}_{T} \mid h_{t}, u_{t}\right)}{f\left(\mathbf{Y}_{T}\right)} d u_{t} d h_{t}
$$

$$
\hat{u}_{t}\left(\mathbf{Y}_{T}\right)=E\left(u_{t} \mid \mathbf{Y}_{T}\right)=\iint u_{t} \frac{f\left(\mathbf{Y}_{t}, h_{t}, u_{t}\right) f\left(\mathbf{y}_{t+1}, \ldots, \mathbf{y}_{T} \mid h_{t}, u_{t}\right)}{f\left(\mathbf{Y}_{T}\right)} d h_{t} d u_{t} .
$$

In both expressions, the density $f\left(\mathbf{Y}_{T}\right)$ is approximated through the marginal likelihood $\tilde{L}(\boldsymbol{\theta} \mid \mathbf{y})$ of Eq. (3) and $f\left(\mathbf{Y}_{t}, h_{t}, u_{t}\right)$ through $l_{t i j}$. The intermediate conditional probabilities, $f\left(\mathbf{y}_{t+1}, \ldots, \mathbf{y}_{T} \mid h_{t}, u_{t}\right)$ can be obtained by using the following backward recursion

1. Define $\mathbf{b}_{T}=\mathbf{1}_{n_{h} n_{u}}$. 
2. Compute the backward conditional probability at time $t$, with $t=$ $T-1, \ldots, 1$ :

$$
\mathbf{b}_{t}=\left(\frac{b-a}{2}\right)\left(\frac{e-d}{2}\right) \operatorname{diag}\left(\mathbf{f}_{t+1}\right)\left(\left(\mathbf{F}_{h} \circ \mathbf{W}_{h}\right) \otimes\left(\mathbf{F}_{u} \circ \mathbf{W}_{u}\right)\right) \mathbf{b}_{t+1}
$$

where the generic element is $b_{t i j}=f\left(\mathbf{y}_{t+1}, \ldots, \mathbf{y}_{T} \mid u_{t}=u_{i}^{*}, h_{t}=h_{j}^{*}\right)$.

With this further recursion, the smoothed values are approximated as

$$
\hat{h}_{t}\left(\mathbf{Y}_{T}\right) \simeq \frac{\sum_{j}^{n_{h}} h_{j}^{*} \sum_{i}^{n_{u}} l_{t i j} b_{t i j}}{\tilde{L}(\boldsymbol{\theta} \mid \mathbf{y})} ; \quad \hat{u}_{t}\left(\mathbf{Y}_{T}\right) \simeq \frac{\sum_{i}^{n_{u}} u_{i}^{*} \sum_{j}^{n_{h}} l_{t i j} b_{t i j}}{\tilde{L}(\boldsymbol{\theta} \mid \mathbf{y})}
$$

2.3. Prediction. The one-step-ahead predicted values of volatility and random effects are derived through the following approximations:

$$
\begin{aligned}
\hat{h}_{t}\left(Y_{t-1}\right) & =E\left(h_{t} \mid Y_{t-1}\right)=\frac{\int h_{t} f\left(h_{t} \mid Y_{t-1}\right) f\left(Y_{t-1}\right) d h_{t}}{\iint f\left(Y_{t-1}, u_{t-1}, h_{t-1}\right) d h_{t-1} d u_{t-1}} \\
& =\frac{\int h_{t}\left\{\int f\left(h_{t} \mid h_{t-1}\right)\left[\int f\left(Y_{t-1}, u_{t-1}, h_{t-1}\right) d u_{t-1}\right] d h_{t-1}\right\} d h_{t}}{\iint f\left(Y_{t-1}, u_{t-1}, h_{t-1}\right) d u_{t-1} d h_{t-1}} \\
& \simeq \frac{\frac{e-d}{2} \sum_{j}^{n_{h}} h_{j} w_{h j} \sum_{j^{\prime}}^{n_{h}} F_{h j j^{\prime}} w_{h j^{\prime}} \sum_{i}^{n_{u}} l_{t-1 i j^{\prime}}}{\sum_{i}^{n_{u}} \sum_{j}^{n_{h}} l_{t-1, i j}} \\
\hat{u}_{t}\left(Y_{t-1}\right) & \simeq \frac{\frac{b-a}{2} \sum_{i}^{n_{u}} u_{i} w_{u i} \sum_{i^{\prime}}^{n_{u}} F_{u i i^{\prime}} w_{u i^{\prime}} \sum_{j}^{n_{h}} l_{t-1, i^{\prime} j}}{\sum_{i}^{n_{u}} \sum_{j}^{n_{h}} l_{t-1, i j}}
\end{aligned}
$$

where $F_{u i i^{\prime}}$ and $F_{h j j^{\prime}}$ denote the generic elements of the transition matrices $F_{u}$ and $F_{h}$ respectively.

3. Application to Tribal Art prices: full table of the estimates. In Table 1 we report the full table that contains the parameter estimates of the three models: fixed effects (FE), autoregressive random effects (ARE) and stochastic volatility with autoregressive random effects (SVARE). For each categorical variable we have indicated the baseline category. The standard errors are in parentheses.

Table 1: Parameter estimates for models FE, ARE and SVARE. Standard errors in parentheses.

\begin{tabular}{lrrr}
\hline & FE & ARE & SVARE \\
\hline$\sigma$ & $1.073(0.006)$ & $1.074(0.008)$ & - \\
$\sigma_{\eta}$ & - & $0.326(0.057)$ & $0.324(0.044)$ \\
$\rho$ & - & $0.833(0.148)$ & $0.862(0.084)$
\end{tabular}


$\alpha$

$\delta$

$\sigma_{\nu}$

$\beta_{0}$

Type of object: baseline Furniture

- Sticks

- Masks

- Religious objects

- Ornaments

- Sculptures

- Musical instruments

- Tools

- Clothing

- Textiles

- Weapons

- Jewels

Material: baseline Ivory

- Vegetable fibre

- Wood

- Metal

- Gold

- Stone

- Terracotta, ceramic

- Silver

- Seashell

- Bone, horn

- Not indicated

Patina: baseline Not indicated

- Pejorative

- Present

- Appreciative

Region: baseline Central Africa

- Southern Africa

- Western Africa

- Eastern Africa

- Australia

- Indonesia

- Melanesia

- Polynesia

- Northern America

- Northern Africa

- Southern America

- Mesoamerica

- Far East

- Micronesia

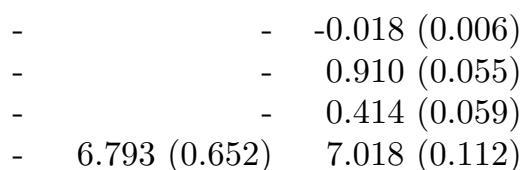

$-0.143(0.069)$

$-0.144(0.075) \quad-0.238(0.064)$

$0.269(0.063) \quad 0.253(0.052)$

$0.053(0.061) \quad 0.051(0.071) \quad 0.038(0.057)$

$-0.306(0.063) \quad-0.306(0.074) \quad-0.232(0.058)$

$0.149(0.054) \quad 0.147(0.058) \quad 0.142(0.049)$

$-0.245(0.083) \quad-0.246(0.105) \quad-0.176(0.076)$

$-0.173(0.055) \quad-0.175(0.062) \quad-0.167(0.050)$

$-0.129(0.093) \quad-0.131(0.107) \quad-0.090(0.080)$

$-0.015(0.095) \quad-0.017(0.098) \quad 0.075(0.082)$

$-0.240(0.069) \quad-0.240(0.074) \quad-0.212(0.065)$

$0.006(0.086) \quad 0.005(0.103) \quad-0.040(0.083)$

$-0.062(0.071) \quad-0.061(0.082) \quad-0.131(0.065)$

$0.279(0.054) \quad 0.280(0.065) \quad 0.194(0.051)$

$-0.151(0.070) \quad-0.152(0.092) \quad-0.139(0.067)$

$0.231(0.082) \quad 0.231(0.101) \quad 0.332(0.077)$

$0.174(0.070) \quad 0.175(0.083) \quad 0.145(0.066)$

$0.043(0.068) \quad 0.044(0.078) \quad 0.063(0.063)$

$-0.128(0.136) \quad-0.128(0.130) \quad-0.157(0.125)$

$0.280(0.115) \quad 0.279(0.152) \quad-0.028(0.112)$

$-0.413(0.091) \quad-0.412(0.121) \quad-0.344(0.089)$

$0.092(0.125) \quad 0.091(0.132) \quad 0.015(0.102)$

$0.446(0.104) \quad 0.445(0.106) \quad 0.365(0.104)$

$-0.003(0.029) \quad-0.004(0.033) \quad-0.035(0.027)$

$0.183(0.029) \quad 0.182(0.035) \quad 0.149(0.028)$

$-0.473(0.075) \quad-0.474(0.105) \quad-0.462(0.074)$

$-0.273(0.029) \quad-0.274(0.032) \quad-0.278(0.026)$

$-0.355(0.071) \quad-0.355(0.083) \quad-0.382(0.065)$

$0.117(0.114) \quad 0.116(0.138) \quad 0.060(0.102)$

$-0.359(0.071) \quad-0.361(0.073) \quad-0.323(0.069)$

$0.002(0.038) \quad 0.001(0.042) \quad-0.031(0.035)$

$0.477(0.045) \quad 0.477(0.053) \quad 0.441(0.041)$

$0.525(0.047) \quad 0.525(0.055) \quad 0.418(0.042)$

$-0.509(0.301) \quad-0.512(0.438) \quad-0.576(0.299)$

$-0.004(0.057) \quad-0.005(0.063) \quad-0.057(0.052)$

$0.245(0.053) \quad 0.243(0.061) \quad 0.155(0.047)$

$-0.499(0.345) \quad-0.500(0.470) \quad-0.155(0.365)$

$0.294(0.155) \quad 0.294(0.194) \quad 0.234(0.142)$ 
- Indian Subcontinent

- Southeastern Asia

- Middle East

Illustration width: baseline Absent

- Col. miscellaneous

- Col. quarter page

- Col. half page

- Col. full page

- Col. more than one

- Col. cover

- b/w miscellaneous

- b/w quarter page

- b/w half page

- b/w full page

- b/w more than one

Description on the cata

- Short visual descr.

- Visual descr.

- Broad visual descr.

- Critical descr.

- Broad critical descr.

CABS (Yes vs No)

CABC (Yes vs No)

CAES (Yes vs No)

Historicization: baseline Absent

- Simple certification

- Museum certification

- Relevant museum certification

Paris (vs New York)

Christie's (vs Sotheby's)

$\begin{array}{rrr}0.824(0.285) & 0.815(0.283) & 0.760(0.242) \\ -0.139(0.343) & -0.146(0.345) & -0.195(0.341) \\ -0.710(0.623) & -0.713(0.701) & -0.661(0.623) \\ & & \\ 0.979(0.048) & 0.980(0.066) & 0.964(0.046) \\ 1.825(0.048) & 1.825(0.063) & 1.567(0.046) \\ 2.224(0.056) & 2.223(0.071) & 1.943(0.053) \\ 2.564(0.060) & 2.565(0.077) & 2.319(0.056) \\ 3.060(0.065) & 3.063(0.086) & 2.857(0.061) \\ 3.375(0.171) & 3.376(0.242) & 3.122(0.168) \\ 1.116(0.107) & 1.114(0.092) & 0.920(0.085) \\ 0.834(0.067) & 0.835(0.070) & 0.673(0.057) \\ 1.403(0.138) & 1.403(0.129) & 1.223(0.106) \\ 2.204(0.542) & 2.204(0.642) & 1.876(0.431) \\ 0.650(0.542) & 0.655(0.389) & 0.471(0.426)\end{array}$

line Absent

$-0.182(0.085)$

$0.237(0.087)$

$0.784(0.094)$

$-0.184(0.102)$

$0.234(0.103)$

$0.784(0.110)$

$0.772(0.113)$

$1.666(0.120)$

$0.248(0.033)$

$0.332(0.027)$

$0.205(0.038)$

$-0.257(0.076)$

$0.152(0.077)$

$0.675(0.084)$

$0.652(0.084)$

$1.487(0.092)$

$0.227(0.028)$

$0.306(0.022)$

$0.205(0.030)$

$0.204(0.034)$

$0.083(0.029)$

$0.102(0.023)$

$0.008(0.036)$

$\begin{array}{rr}0.104(0.041) & 0.062(0.032) \\ -0.302(0.035) & -0.197(0.026)\end{array}$

$-0.255(0.030) \quad-0.335(0.025)$ 
4. Application to Tribal Art prices: choice of the quadrature based method. In Table 2 we report the results of the estimation of the SVARE model under the Gauss-Legendre (GL) and the rectangular quadrature (RQ) method. We allow the quadrature points to vary from 21 to 61 . In particular, we refer to the following sets: $\left(n_{u}=21, n_{h}=21\right),\left(n_{u}=31, n_{h}=\right.$ $31),\left(n_{u}=41, n_{h}=41\right),\left(n_{u}=51, n_{h}=51\right)$, and $\left(n_{u}=61, n_{h}=61\right)$.

TABLE 2

Comparison of $G L$ and $R Q$ with different numbers of quadrature points

\begin{tabular}{rccccrrrrrrr}
\hline & $n_{u}$ & $\beta_{0}$ & $\rho$ & $\sigma_{\eta}$ & $\alpha$ & $\delta$ & $\sigma_{\nu}$ & $\log$-lik & $\begin{array}{r}\text { time } \\
\text { (hours) }\end{array}$ & $\begin{array}{r}\text { no. of } \\
\text { iter. }\end{array}$ & CV \\
\hline \multirow{4}{*}{ GL } & 21 & 7.761 & 0.865 & 0.325 & 0.166 & 0.879 & 0.353 & -20190.53 & 4.53 & 78 & 0.724 \\
& 31 & 7.285 & 0.817 & 0.322 & 0.051 & 0.917 & 0.411 & -20176.61 & 12.58 & 66 & 1.888 \\
& 41 & 7.269 & 0.851 & 0.329 & 0.121 & 0.940 & 0.370 & -20171.93 & 32.67 & 71 & 2.229 \\
& 51 & 7.018 & 0.862 & 0.324 & -0.018 & 0.910 & 0.414 & -20167.98 & 66.25 & 75 & 1.727 \\
& 61 & 7.170 & 0.834 & 0.322 & -0.071 & 0.933 & 0.402 & -20170.24 & 114.38 & 72 & 2.457 \\
\hline \multirow{6}{*}{ RQ } & 21 & 7.133 & 0.811 & 0.323 & 0.057 & 0.854 & 0.395 & -20167.60 & 4.05 & 71 & 0.780 \\
& 31 & 7.019 & 0.835 & 0.321 & -0.111 & 0.941 & 0.385 & -20170.48 & 13.22 & 71 & 2.644 \\
& 41 & 7.110 & 0.869 & 0.326 & -0.098 & 0.925 & 0.394 & -20170.31 & 32.96 & 73 & 1.936 \\
& 51 & 7.096 & 0.851 & 0.323 & 0.039 & 0.934 & 0.402 & -20170.51 & 65.60 & 71 & 2.571 \\
\hline \multicolumn{7}{c}{ Notes: RQ with 61 quadrature points does not converge. }
\end{tabular}

The two methods perform quite similarly. This is in agreement with the results given in literature for stochastic volatility models (see, for example Cagnone and Bartolucci, 2016), according to which GL and RQ have similar performance when the squared coefficients of variation of the volatility $\mathrm{CV}=\exp \left(\sigma_{\nu}^{2} /\left(1-\delta^{2}\right)\right)-1$ is greater or equal than 1 (see the last column of Table 2). Both methods produce similar estimates with the only exception of $\alpha$. However, the high mean square error of this estimator is quite common in the stochastic volatility models, as highlighted in several simulation studies (see for example Bartolucci and De Luca (2001) and Fridman and Harris (1998)). Also, note that RQ with 61 quadrature points did not reach convergence. Apart from RQ with 21 points, the highest value of the loglikelihood is obtained with GL with 51 points. This is also the solution with the best forecasting performance among the GL results (Table 3). Moreover, 51 points should be enough to ensure an accurate approximation of the standard errors. For all these reasons, we chose GL with 51 points.

5. Application to Tribal Art prices: entropy based diagnostic tests for serial independence and nonlinearity. We study the time dependence of level-2 quantities such as residuals or predicted random effects, by means of the entropy based metric $S_{k}$ under two null hypotheses on the data generating process $\left\{X_{t}\right\}$ :

1. Independence: $H_{0}: X_{t}$ and $X_{t-k}$ are independent, for $k=1, \ldots$, 
TABLE 3

Forecasting performance of GL with different numbers of quadrature points

\begin{tabular}{crrr}
\hline & \multicolumn{3}{c}{ Forecasting } \\
$n_{u}, n_{h}$ & MAE & MAPE & RMSE \\
\hline 21 & 0.835 & 0.076 & 1.156 \\
31 & 0.848 & 0.077 & 1.171 \\
41 & 0.834 & 0.076 & 1.154 \\
51 & 0.826 & 0.076 & 1.146 \\
61 & 0.832 & 0.076 & 1.152 \\
\hline
\end{tabular}

2. Linearity: $H_{0}^{\prime}: X_{t}=\sum_{j=1}^{\infty} \phi_{j} X_{t-j}+\varepsilon_{t}$ with $\left\{\varepsilon_{t}\right\}$ is a (zero mean) i.i.d process

$H_{0}^{\prime}$ is consistent with the formal definition of linear processes. The test statistic $\hat{S}_{k}$ is based on the entropy based measure of dependence $S_{k}$ :

$\hat{S}_{k}=\frac{1}{2} \iint\left[\left(\hat{f}_{\left(X_{t}, X_{t+k}\right)}\left(x_{1}, x_{2}\right)\right)^{1 / 2}-\left(\hat{f}_{X_{t}}\left(x_{1}\right) \hat{f}_{X_{t+k}}\left(x_{2}\right)\right)^{1 / 2}\right]^{2} w\left(x_{1}, x_{2}\right) d x_{1} d x_{2}$

where $w\left(x_{1}, x_{2}\right)$ is a continuous weight function and $\hat{f}_{X_{t}}, \hat{f}_{X_{t+k}}$ and $\hat{f}_{\left(X_{t}, X_{t+k}\right)}$ are the kernel density estimators for $f_{X_{t}}, f_{X_{t+k}}$ and $f_{\left(X_{t}, X_{t+k}\right)}$ respectively. The distribution of $\hat{S}_{k}$ under the null hypotheses is derived by means of resampling methods, for more details see Granger et al. (2004); Giannerini et al. (2015).

5.1. Random effects model (RE). The random effects model has the following specification

$$
\log \left(y_{i t}\right)=\beta_{0}+u_{t}+\mathbf{x}_{i t}^{\prime} \boldsymbol{\beta}+\epsilon_{i t}, \quad \epsilon_{i t} \mid \mathbf{x}_{t} \sim \operatorname{NID}\left(0, \sigma^{2}\right)
$$

we apply the diagnostic tests to $\hat{u}_{t}$, the best linear unbiased predictor (BLUP) of the random effect $u_{t}$. 

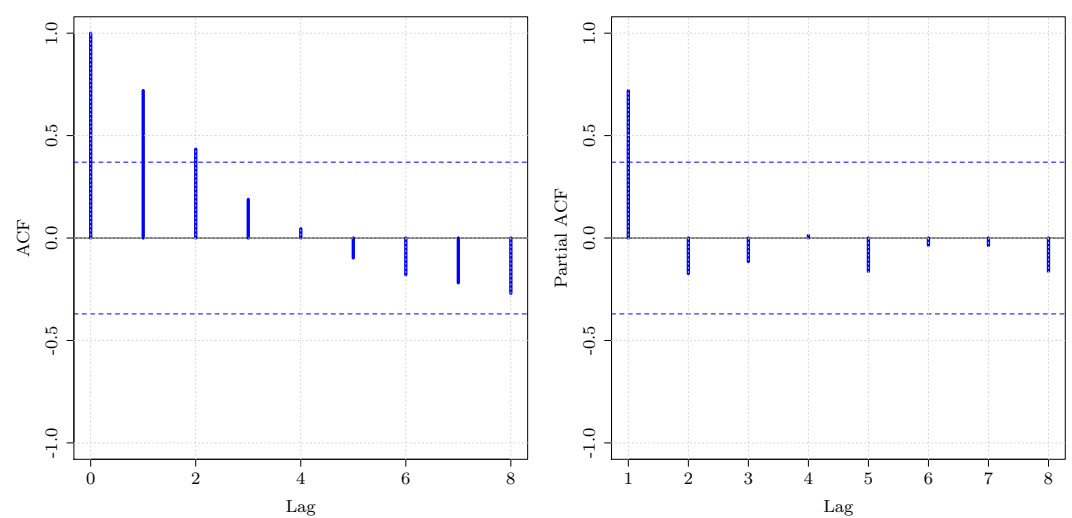

FIG 1. ACF (left) and PACF (right) of the predicted random effects $\hat{u}_{t}$ for the random effects model (RE).

The results point to a $\mathrm{AR}(1)$ structure.
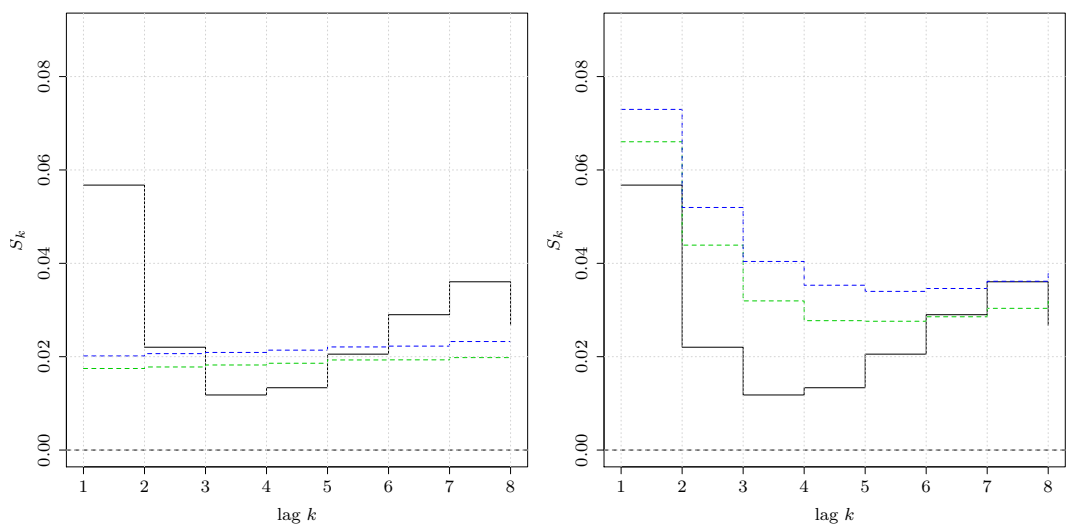

FIG 2. Entropy measure of dependence of the predicted random effects $\hat{u}_{t}$ for the random effects model (RE). (left) Serial independence and non linear serial dependence (right)

The results confirm the existence of a significant linear dependence structure with no evidence of nonlinear effects.

5.2. Autoregressive Random effects model (ARE). The model has the following specification

$$
\begin{array}{rlrl}
\log \left(y_{i t}\right) & =\beta_{0}+u_{t}+\mathbf{x}_{i t}^{\prime} \boldsymbol{\beta}+\epsilon_{i t}, & \epsilon_{i t} \mid \mathbf{x}_{t} \sim \operatorname{NID}\left(0, \sigma^{2}\right) \\
u_{t} & =\rho u_{t-1}+\eta_{t}, & \eta_{t} \mid \mathbf{x}_{t} & \sim \operatorname{NID}\left(0, \sigma_{\eta}^{2}\right)
\end{array}
$$


first, we apply the diagnostic tests to level-2 residuals $\hat{\eta}_{t}$ :
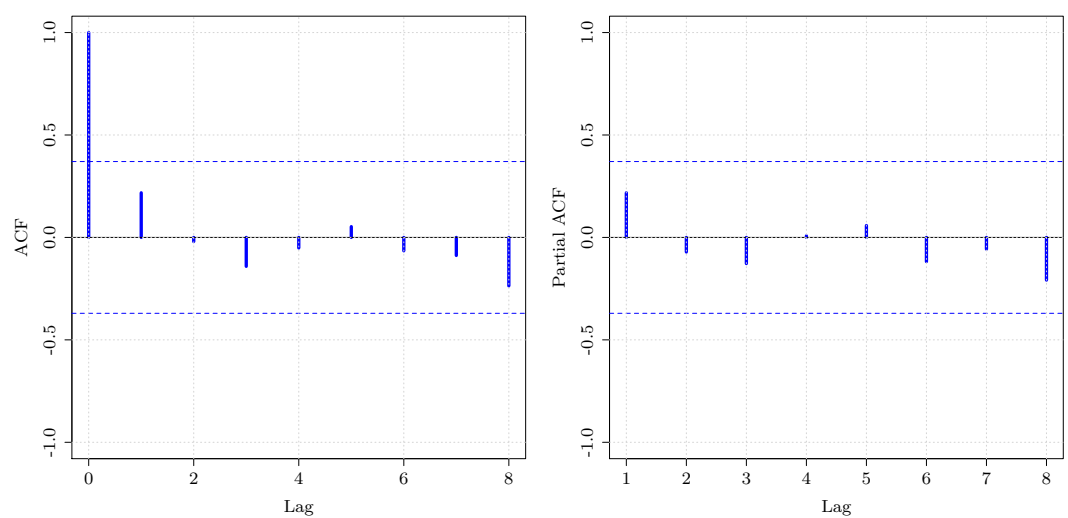

FIG 3. ACF (left) and PACF (right) of level-2 residuals $\hat{\eta}_{t}$ for the ARE model.

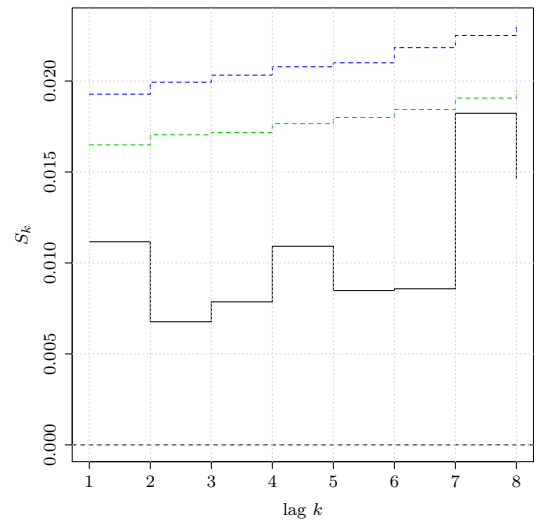

FIG 4. Entropy measure of dependence of level-2 residuals $\hat{\eta}_{t}$ for the ARE model.

The results indicate that no residual dependence is present in $\hat{\eta}_{t}$. Second, we study $s_{t}^{\mathrm{ARE}}$ the standard deviation of level-1 residuals $\hat{\epsilon}_{i t}$. 

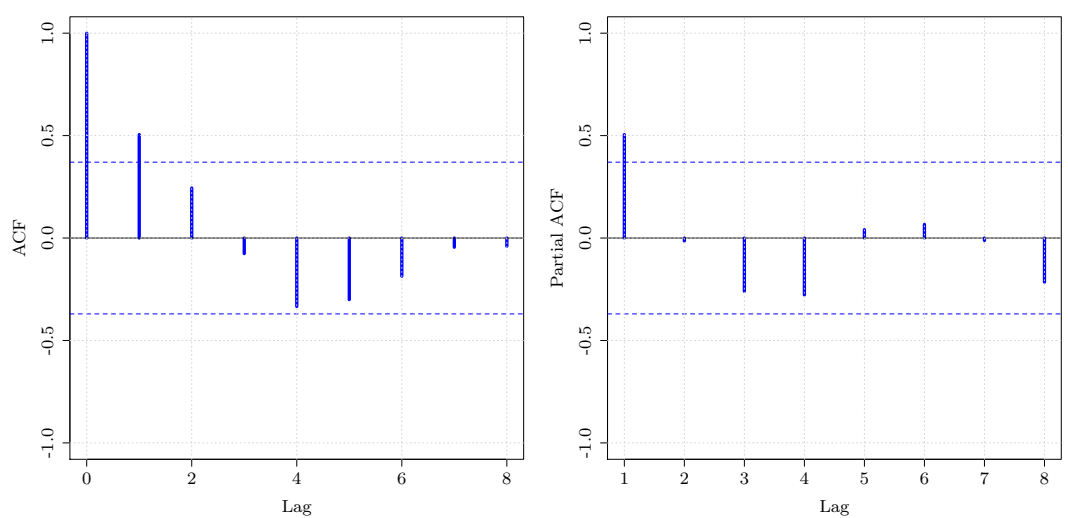

FIG 5. ACF (left) and PACF (right) of $s_{t}^{A R E}$, the standard deviations of level-1 residuals for the ARE model.
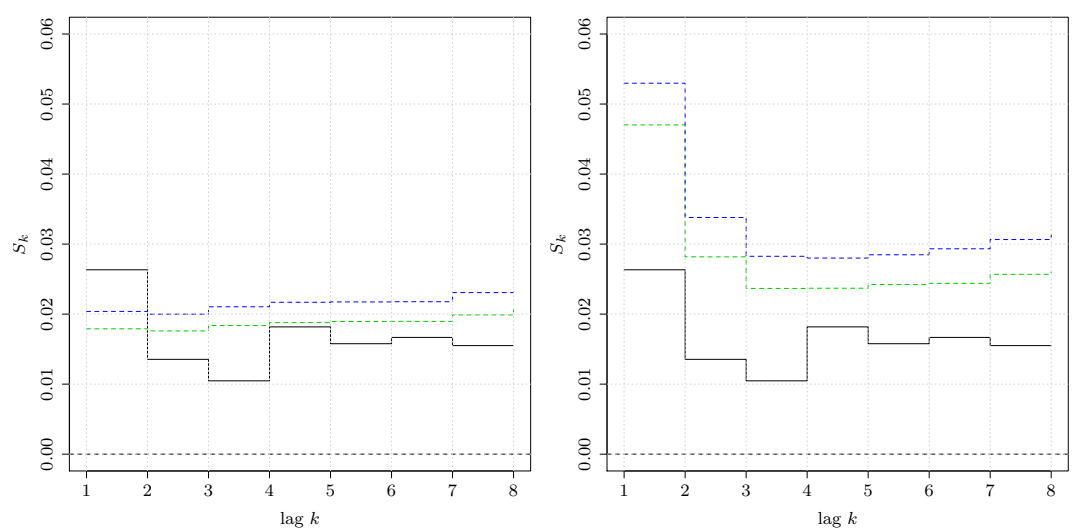

FIG 6. Entropy measure of dependence of $s_{t}^{A R E}$, the standard deviations of level-1 residuals for the ARE model. (left) Serial independence; (right) non linear serial dependence.

The results point to a linear $\mathrm{AR}(1)$ structure for the volatility.

5.3. Stochastic Volatility Autoregressive Random effects model (SVARE). The model has the following specification

$$
\begin{aligned}
\log \left(y_{i t}\right) & =\beta_{0}+u_{t}+\mathbf{x}_{i t}^{\prime} \boldsymbol{\beta}+\epsilon_{i t}, & & \epsilon_{i t} \mid \mathbf{x}_{t} \sim \operatorname{NID}(0,1) \\
u_{t} & =\rho u_{t-1}+\eta_{t}, & \eta_{t} \mid \mathbf{x}_{t} & \sim \operatorname{NID}\left(0, \sigma_{\eta}^{2}\right) \\
h_{t} & =\alpha+\delta h_{t-1}+\sigma_{\nu} \nu_{t}, & \nu_{t} \mid \mathbf{x}_{t} & \sim \operatorname{NID}(0,1)
\end{aligned}
$$

first, we apply the diagnostic tests to $s_{t}^{\mathrm{SV}}$ the standard deviations of level-1 residuals $\hat{\epsilon}_{i t}$. 

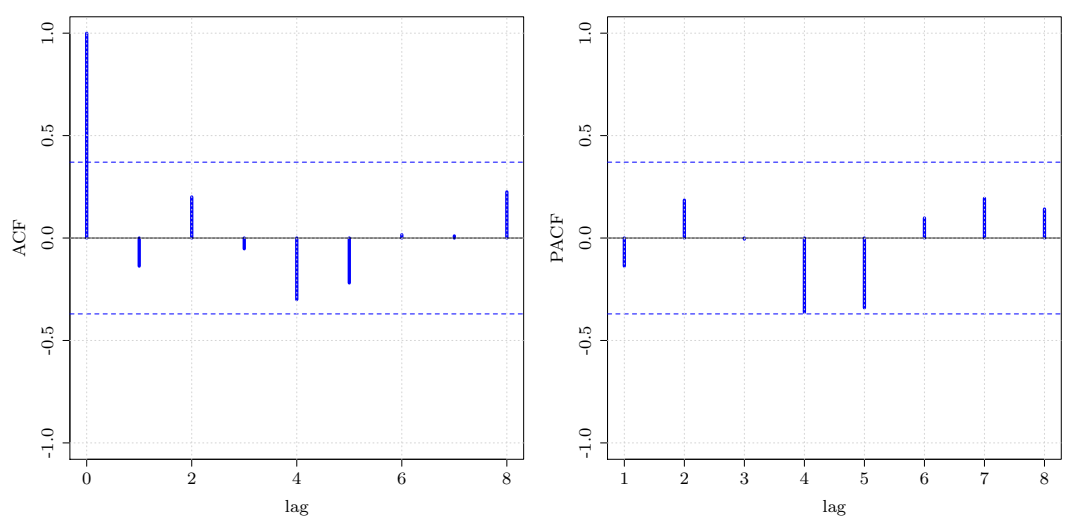

FIG 7. ACF (left) and PACF (right) of $s_{t}^{S V}$ the standard deviations of level-1 residuals for the SVARE model.

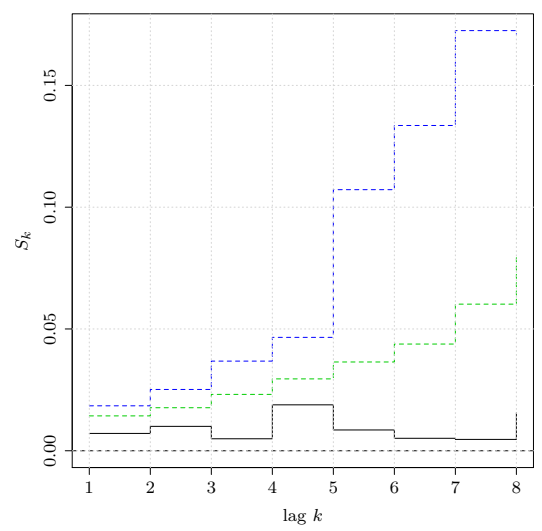

FIG 8. Entropy measure of dependence for $s_{t}^{S V}$ the standard deviations of level-1 residuals for the SVARE model.

there is no residual dependence in the volatility.

Second, we apply the diagnostic tests to level-2 residuals $\hat{\nu}_{t}$ : 

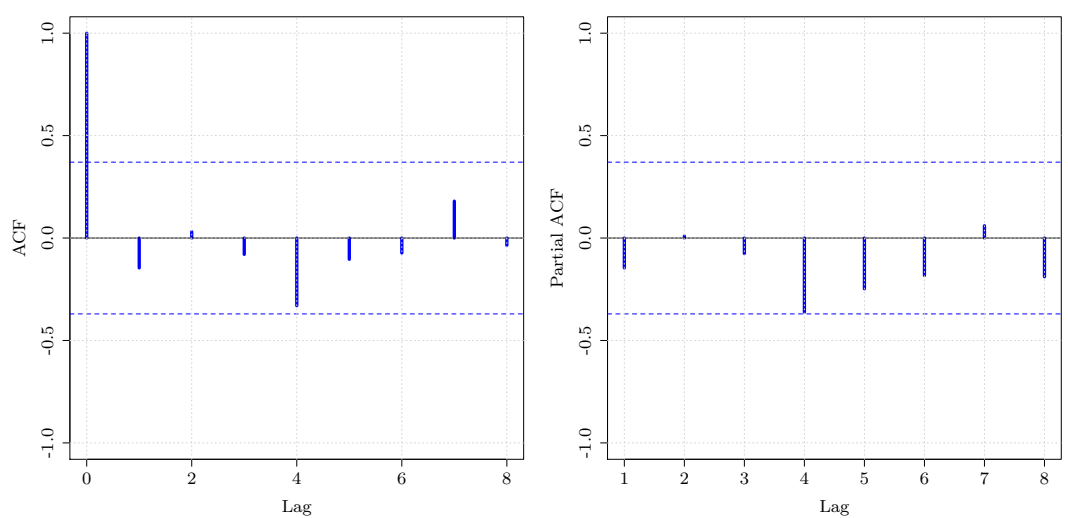

FIG 9. ACF (left) and PACF (right) of level-2 residuals $\hat{\nu}_{t}$ of the SVARE model.

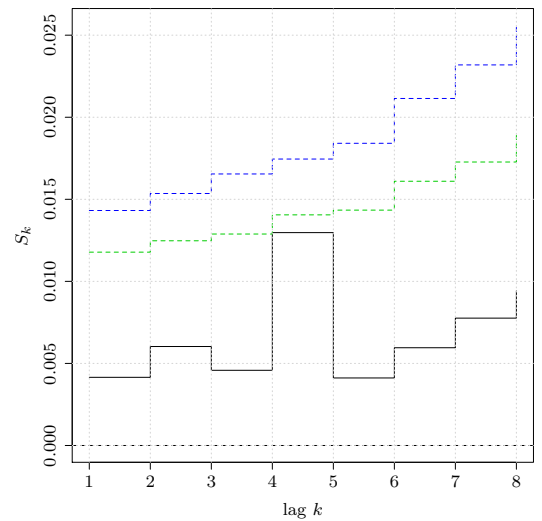

FIG 10. Entropy measure of dependence of level-2 residuals $\hat{\nu}_{t}$ of the SVARE model.

Again, there is no evidence of residual dependence.

The following plots compare the behaviour of level-1 residuals for the models. 

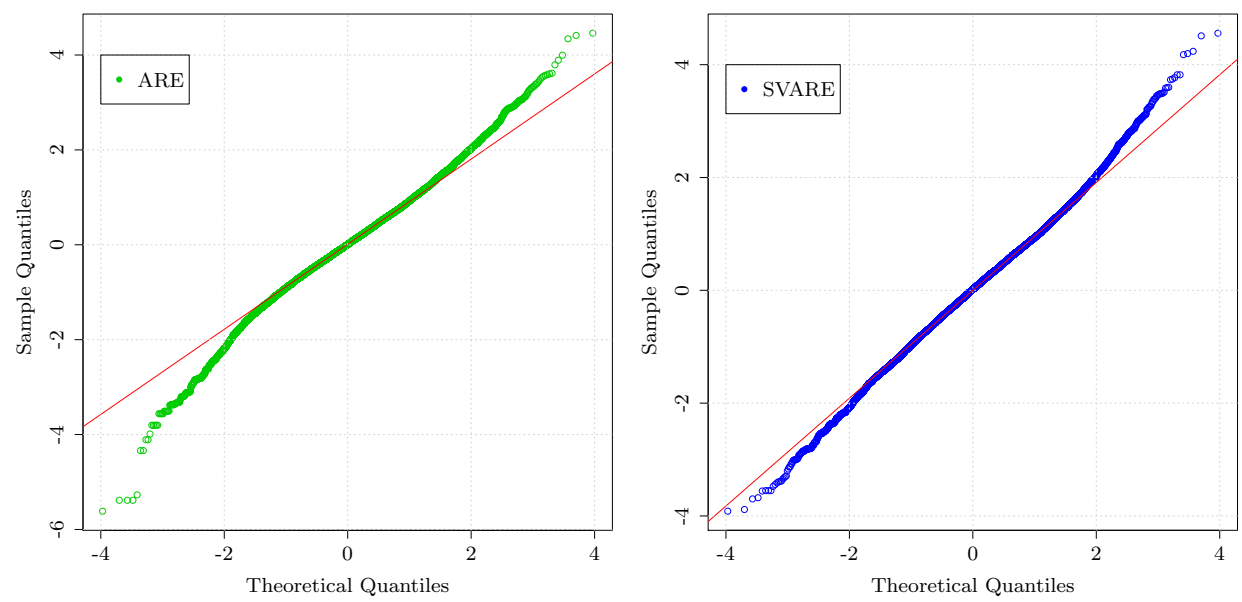

FIG 11. qqplot of level-1 residuals for the ARE (left) and the SVARE (right) models.

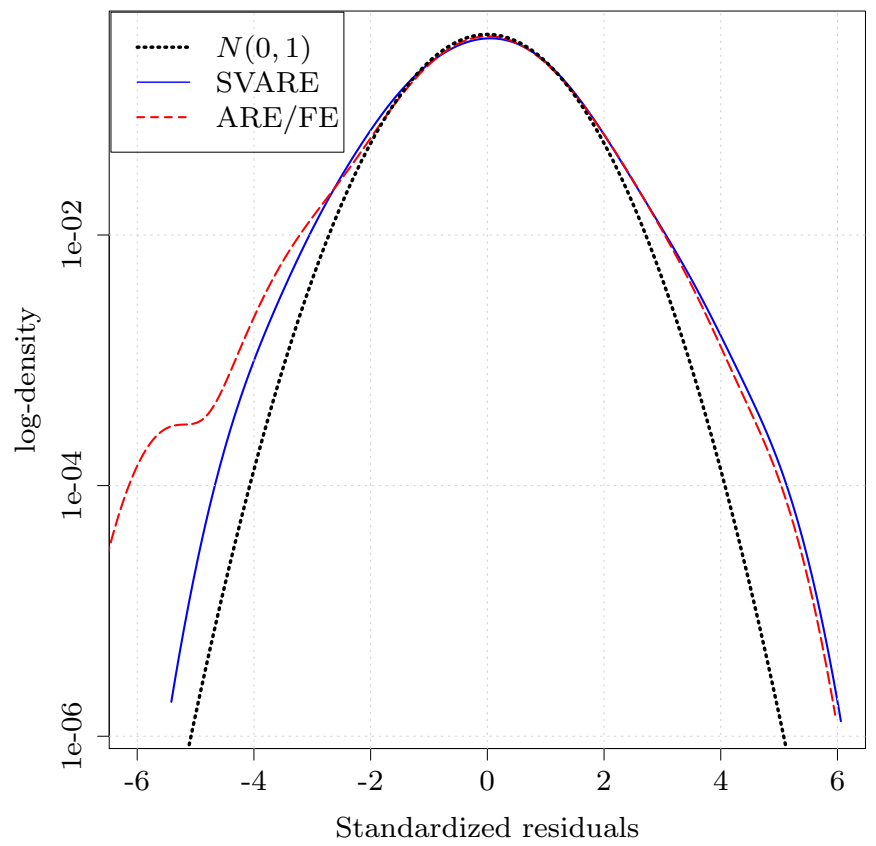

FIG 12. log-density of level-1 residuals for the FE/ARE and the SVARE models.

The log-density plot of level-1 residuals highlights the non-normality in the tails of the densities. 
6. Software implementation. The software infrastructure has been implemented by using pure R (R Core Team, 2016) mixed with compiled code in the form of shared libraries for the most demanding tasks. In particular, the fixed effects model (FE) has been estimated through the glm function. As for the Autoregressive Random Effects model (ARE) we have implemented different routines in pure R: the EM algorithm for parameter estimation, the RQ and GL quadrature methods for filtering and prediction, the wild bootstrap for standard errors. Due to the high computational burden, most of the routines for the SVARE model have been coded in fortran shared libraries embedded within $\mathrm{R}$. The minimization of the log-likelihood function has been performed through the unconstrained BFGS method available in the optim function. The diagnostic tests are implemented in the package tseriesEntropy (Giannerini, 2015). The R package multilevelRCS that includes the implementation of the ARE and SVARE models is forthcoming.

\section{References.}

Bartolucci, F. and G. De Luca (2001). Maximum likelihood estimation of a latent variable time-series model. Applied Stochastic Models in Business and Industry 17, 5-17.

Cagnone, S. and F. Bartolucci (2016). Adaptive quadrature for maximum likelihood estimation of a class of dynamic latent variable models. Computational Economics in press, $1-24$.

Fridman, M. and L. Harris (1998). A Maximum Likelihood Approach for Non-Gaussian Stochastic Volatility Models. Journal of Business 8 Economic Statistics 16(3), 284291.

Giannerini, S. (2015). tseriesEntropy: Entropy Based Analysis and Tests for Time Series. $\mathrm{R}$ package version $0.5-13$.

Giannerini, S., E. Maasoumi, and E. Bee Dagum (2015). Entropy testing for nonlinear serial dependence in time series. Biometrika 102, 661-675.

Granger, C., E. Maasoumi, and J. Racine (2004). A dependence metric for possibly nonlinear processes. J. Time Ser. Anal. 25(5), 649-669.

Harvey, A., E. Ruiz, and N. Shephard (1994). Multivariate stochastic variance models. The Review of Economic Studies 61(2), pp. 247-264.

R Core Team (2016). R: A Language and Environment for Statistical Computing. Vienna, Austria: R Foundation for Statistical Computing.

Searle, S., G. Casella, and C. McCulloch (1992). Variance components. New York: Wiley.

Skrondal, A. and S. Rabe-Hesketh (2004). Generalized latent variable modeling: multilevel, longitudinal, and structural equation models. New York: Chapman \& Hall/CRC.

Dipartimento di Scienze Statistiche

Via delle Belle arti 41, 40126, Bologna, Italy.

E-MAIL: silvia.cagnone@unibo.it

simone.giannerini@unibo.it

lucia.modugno@unibo.it 